\section{A Simple Modification of Morse's Rule}

MoRsE $^{1}$ introduced an empirical rule to the effect that

$$
\omega_{e} r_{e}^{3} \approx 3 \times 10^{-21} \mathrm{~cm}^{2} \ldots \ldots \ldots(1)
$$

where $\omega_{e}, r_{e}$, respectively, are the equilibrium nuclear vibration frequency (in $\mathrm{cm}^{-1}$ ) and the equilibrium nuclear separation (in $\mathrm{cm}$.) of a diatomic molecule, as deduced from spectra. In a recent paper $^{2}$, dealing with the classification of non-hydride diatomic molecules into groups and periods, I have emphasised the importance of the 'group number' $n$, equal to the number of 'shared' electrons, or total number of 'valency' electrons of the two separate atoms. The way in which the errors from the strict requirements of Morse's rule distribute themselves in certain periods suggests that the insertion of some function of the group number into the Morse expression might lead to better agreement with observation. For non-hydride diatomic molecules of the period containing two completed $K$ rings associated with each nucleus, I have derived the following empirical modification of Morse's relationship :

$$
\omega_{e} r_{e}^{3} \sqrt{n}=9.55 \times 10^{-21} \mathrm{~cm}^{2} \quad \ldots(2)
$$

The mean error in deduction of $r_{e}$ from $\omega_{e}$ values for 29 test cases of electronic levels of diatomic molecules of the specified kind amounts to $\pm 1 \cdot 3$ per cent from experimental values, whilst the mean error using the unmodified Morse expression for the same cases is $\pm 5 \cdot 2$ per cent. The results will be communicated in due course in another place.

C. H. Douglas Clark.

Department of Inorganic Chemistry,

University,

Leeds.

May 4.

'P. M. Morse, Phys. Rev., (ii), 34, 57-64; 1929

2 C. H. Douglas Clark, Proc. Leeds Phil. Soc., 2, 502-512; 1934.

\section{Inheritance in Fresh-water Ostracods}

Prof. MacBride's recent article in NAture ${ }^{1}$, on "Inheritance of Acquired Habits", leads me to direct attention to some interesting information which is available from the study of fresh-water ostracods.

Fresh-water ostracods possess both relatively and absolutely the largest sperms known throughout the animal kingdom, while quite recently it has been discovered that these enormous sperms are highly motile. The sperms are passed into the spermatheca of the female, which possesses a spermathecal duct highly complicated in structure and also exceptionally long. Under the proper conditions, the large sperms can be seen moving very actively both in the spermatheca and also in the upper or proximal region of the duct. The fresh-water ostracods are also remarkable for the wide prevalence of parthenogenesis. In some cases, whole genera exist in which males are unknown. One of the best known genera in this connexion is that of Herpetocypris, containing the well-known species $H$. reptans which abounds practically in every pond in the British Isles and is distributed throughout Europe.

The genus is a well-defined one, and two years ago, taking the genus as described by Sars in "Crustacea of Norway" (vol. 9), I estimated that there were some twelve species occurring throughout the world, and in no case were the males known. The most remarkable fact remains, however, that the spermatheca, and in particular the spermathecal duct, remains in $H$. reptans and in all other species examined, nor does it show the slightest sign of degeneration. It is not proposed to give here further taxonomic details, but anyone familiar with the taxonomy of fresh-water ostracods will know many parallel instances.

It is fairly obvious that at one time the males must have existed in each species of Herpetocypris, and, since the males have disappeared entirely from the genus, exclusive parthenogenetic reproduction must have been going on for a considerable length of time, most probably for thousands if not millions of generations; yet this useless spermathecal duct remains.

If we treat the matter from a genetical point of view there is a fairly simple explanation, but it seems to me extremely difficult to account for the persistence of this highly complicated genital organ if we accept the theory of the "Inheritance of Acquired Habit". Moreover, the case becomes all the more striking when we consider other groups of animals in which parthenogenesis occurs. For here it is almost universally true that individuals reproducing solely by parthenogenesis usually have their genital organs impaired in some way.

\section{Marlborough College.}

A. G. Lowndes.

${ }^{2}$ NATURE, 133, 598, April 21, 1934.

Mr. Lowndes has misunderstood my article. Its purpose was not to put forward a theory of the heritability of acquired habit but to show that this heritability has been experimentally proved to be a fact. If this is so, it is possible to explain all cases where the course of evolution has been followed in detail, as well as to explain the recapitulatory element in development.

E. W. MacBride.

Imperial College of Science, London, S.W.7.

\section{Parasitism in Heavy Water of Low Concentration}

THe first biological experiments ${ }^{1}$ with heavy water (May 1933) showed that a low concentration of diplogen ( 1 part in 2,000) may have a beneficial effect on forms such as Spirogyra (the average longevity of 355 cells, in filament sections of 10-50 cells, in the diplogen water was 7.6 days, and the average for 322 cells in ordinary water was $1 \cdot 6$ days). It was also reported ${ }^{2}$ that cell division in Euglena is increased in this dilute heavy water (density 1.00006). Meyer ${ }^{3}$ confirmed the dilute heavy water effect by demonstrating that mats of Aspergillus showed sixteen times the dry weight of controls.

We have found that flatworms (Planaria maculata and Phagocata gracilis) kept in dilute heavy water for long periods show a striking difference in the rate of shrinkage in body size. After four months, the animals in ordinary water were only one fifth the length of the specimens in the diplogen water. This was probably due to reduced enzymic hydrolysis in the starving animals, since we have shown ${ }^{4}$ that the dilute heavy water reduces the activity of amylase and zymin (the enzyme and substrate were incubated separately in the water and no effect was 